\title{
$t$-perfection is always strong for claw-free graphs
}

\author{
Henning Bruhn Maya Stein*
}

\begin{abstract}
A connected graph $G$ is called $t$-perfect if its stable set polytope is determined by the non-negativity, edge and odd-cycle inequalities. Moreover, $G$ is called strongly $t$-perfect if this system is totally dual integral. It is an open problem whether $t$-perfection is equivalent to strong $t$-perfection. We prove the equivalence for the class of claw-free graphs.
\end{abstract}

\section{Introduction}

For a graph $G=(V, E)$ define the polytope $\operatorname{TSTAB}(G)$ as the set of all vectors $x \in \mathbb{R}^{V}$ satisfying

$$
\begin{aligned}
0 \leq x_{v} \leq 1 & \text { for every vertex } v \in V, \\
x_{u}+x_{v} \leq 1 & \text { for every edge } u v \in E, \\
\sum_{v \in V(C)} x_{v} \leq\left\lfloor\frac{|V(C)|}{2}\right\rfloor & \text { for every odd cycle } C \text { in } G .
\end{aligned}
$$

The graph $G$ is called $t$-perfect if $\operatorname{TSTAB}(G)$ coincides with the stable set polytope of $G$ (the convex hull of characteristic vectors of stable sets in $\mathbb{R}^{V}$ ). We call $G$ strongly t-perfect if the system (1) is totally dual integral (TDI). Hence, by definition strong $t$-perfection implies $t$-perfection. Whether the converse is true is not known.

Question 1. Is every t-perfect graph also strongly t-perfect?

The question is briefly discussed in Schrijver [14, Vol. B, Ch. 68], where also more details about strong and ordinary $t$-perfection can be found. Our main theorem, which we prove in Section 4, answers Question 1 affirmatively for claw-free graphs. (A graph is claw-free if it does not contain $K_{1,3}$ as an induced subgraph.)

Theorem 2. A claw-free graph is t-perfect if and only if it is strongly t-perfect.

The class of $t$-perfect graphs has been introduced by Chvàtal [3], and has been studied by Sbihi and Uhry [12], Shepherd [15], and Gerards and Shepherd [8], among others. Recently, we characterised claw-free $t$-perfect graphs in terms of forbidden substructures, see [2]. Strongly $t$-perfect graphs have been investigated by Gerards [7] and Schrijver [13].

*The second author has been supported by Fondecyt grant no. 11090141. 
The class of strongly $t$-perfect graphs includes bipartite, as well as almost bipartite graphs, that is, graphs whose odd cycles all share a common vertex. A graph that is neither $t$-perfect nor strongly $t$-perfect is $K_{4}$. The first can be seen by considering the vector with value $\frac{1}{3}$ at every vertex of $K_{4}$. This vector belongs to $\operatorname{TSTAB}\left(K_{4}\right)$ but not to its stable set polytope. On the other hand, the weight vector $w_{v}=1$ for every $v \in V\left(K_{4}\right)$ is a witness for the strong $t$-imperfection of $K_{4}$.

The $K_{4}$ is also at the heart of the most wide-reaching certificate for strong $t$-perfection known so far. Call a subdivision of $K_{4}$ odd if every triangle of $K_{4}$ becomes an odd cycle in the subdivision.

Theorem 3 (Gerards [7]). A graph without an odd- $K_{4}$-subdivision as a subgraph is strongly t-perfect.

The theorem has been strengthened by Schrijver in [13]. See also Gijswijt and Schrijver [9] for a more general result.

As throughout the paper, the cycles and their squares will play a prominent role, let us quickly fix some notation in that respect. The (induced) cycle of length $i$ is denoted $C_{i}$, and we assume $C_{i}$ to have vertices $\left\{v_{1}, \ldots, v_{i}\right\}$ occurring in this order on $C_{i}$. The square of $C_{i}$ is $C_{i}^{2}$, and it is obtained from $C_{i}$ by adding an edge between any two vertices of distance 2 .

All graphs in this paper are finite and simple. For general graph theoretical notation and concepts, we refer to Diestel [5].

\section{$2 t$-minors and strong $t$-perfection}

We start by giving an alternative, and sometimes more convenient, definition of strong $t$-perfection. Then, we will describe substructures that are compatible with strong $t$-perfection.

Let $G$ be a graph and let $\mathcal{K}=\mathcal{V} \cup \mathcal{E} \cup \mathcal{C}$ be a family of vertices ( $($ set $\mathcal{E}$ ) and odd cycles $(\operatorname{set} \mathcal{C}$ ) of $G$. We say $\mathcal{K}$ has cost

$$
|\mathcal{V}|+|\mathcal{E}|+\sum_{C \in \mathcal{C}} \frac{|V(C)|-1}{2} .
$$

We say that $\mathcal{K}$ covers a vertex $v k$ times if $v$ lies in at least $k$ members of $\mathcal{K}$. For a weight function $w \in \mathbb{Z}^{V(G)}$, we call $\mathcal{K}$ a $w$-cover of $G$ if every vertex $v$ is covered at least $w_{v}$ times by $\mathcal{K}$.

Observe that every $w$-cover can be turned into an exact cover with the same or lower cost, i.e. into a cover that covers every vertex $v$ exactly $w_{v}$ times (provided $w \geq 0$ ). Indeed, this can easily be achieved by replacing odd cycles incident with an overly covered vertex $v$ by a maximal matching of the cycle that misses $v$, by replacing incident edges by the other endvertex and/or by omitting $v$ itself from the cover, if present.

Furthermore, we can usually assume that any cycle in a $w$-cover is induced. To achieve this, suppose a $w$-cover $\mathcal{K}$ contains an odd cycle $C$ that has a chord. Then $E(C)+e$ decomposes into the edge set of an odd cycle $C^{\prime}$ through $e$ and the edge set of an odd subpath $P$ of $C$ between the endvertices of $e$. Replacing $C$ by $C^{\prime}$ plus every other edge in $P$ yields a cover of the same cost in which every vertex is as often covered as in $\mathcal{K}$. 
For a subset $S$ of $V(G)$, write $w(S):=\sum_{s \in S} w_{s}$, and define the weighted stability number of $G$

$$
\alpha_{w}(G):=\max \{w(S): S \subseteq V(G) \text { is stable }\} .
$$

By linear programming duality, $G$ is strongly $t$-perfect if and only if there is a $w$-cover of cost $\alpha_{w}(G)$ for all $w \in \mathbb{Z}^{V(G)}$, see Schrijver [14] for more details. Moreover, it is easy to see that we need only consider non-negative $w$.

Let $v$ be a vertex of a graph $G$ so that its neighbourhood $N(v)$ forms a stable set. Then $\tilde{G}$ is obtained from $G$ by a $t$-contraction at $v$ if $\tilde{G}=G / E(v)$, i.e. if $\tilde{G}$ is the result of contracting all the edges incident with $v$. We say that $G^{\prime}$ is a $t$-minor of $G$ if $G^{\prime}$ can be obtained from $G$ by a sequence of vertex deletions and $t$-contractions.

It is not hard to check that $t$-perfection is stable under taking $t$-minors. Indeed, verifying that induced subgraphs of $t$-perfect graphs are $t$-perfect is easy, and in Gerards and Shepherd [8] it is shown that if $G^{\prime}$ is obtained from a $t$-perfect graph $G$ by $t$-contraction then $G^{\prime}$ is $t$-perfect, too.

The same holds for strong $t$-perfection: ${ }^{1}$

Proposition 4. Every $t$-minor of a strongly t-perfect graph is strongly t-perfect.

Proof. Let $G$ be strongly $t$-perfect. It is straightforward to see that induced subgraphs of $G$ are strongly $t$-perfect, too. It remains to show, therefore, that for every vertex $v$ with stable neighbourhood $N(v)$ the graph $\tilde{G}:=G / E(v)$ is strongly $t$-perfect as well.

Denote the new vertex of $\tilde{G}$ by $\tilde{v}$. Given a non-negative weight $\tilde{w} \in \mathbb{Z}^{V(\tilde{G})}$, we have to find a $\tilde{w}$-cover $\tilde{K}$ of $\tilde{G}$ that has $\operatorname{cost} \alpha_{\tilde{w}}(G)$.

Set $\beta:=\tilde{w}(V(\tilde{G}))+1$, and define $w \in \mathbb{Z}^{V(G)}$ as $w_{u}:=\tilde{w}_{u}$ for $u \in V(\tilde{G}-\tilde{v})$, $w_{p}:=\beta$ for $p \in N(v)$ and $w_{v}:=d(v) \cdot \beta-\tilde{w}_{\tilde{v}}$. Note that by the choice of $\beta$, every stable set of maximal weight with respect to $w$ either contains $v$, or all of $N(v)$. In either case,

$$
\alpha_{w}(G) \leq \alpha_{\tilde{w}}(\tilde{G})+d(v) \cdot \beta-\tilde{w}_{\tilde{v}}=\alpha_{\tilde{w}}(\tilde{G})+w_{v} .
$$

As $G$ is strongly $t$-perfect, there exists a $w$-cover $\mathcal{K}$ of cost $\alpha_{w}(G)$, which we may assume to cover $v$ exactly $w_{v}$ times. Moreover, we may require all the cycles in $\mathcal{K}$ to be induced.

Let $\mathcal{K}_{v} \subseteq \mathcal{K}$ consist of all $K \in \mathcal{K}$ that are incident with $v$. For each cycle $C \in \mathcal{K}_{v}$ contract the two edges incident with $v$. Note that this gives a cycle in $\tilde{G}$ as $C$ was induced by assumption. Denote the family of the thus obtained cycles by $\tilde{\mathcal{K}}_{v}$. Since we omit all the edges and vertices of $\mathcal{K}_{v}$ in $\tilde{\mathcal{K}}_{v}$, and since every cycle in $\tilde{\mathcal{K}}_{v}$ is two edges shorter than the corresponding cycle in $\mathcal{K}_{v}$, it follows that $\tilde{\mathcal{K}}_{v}$ costs $w_{v}$ less than $\mathcal{K}_{v}$.

Next, we turn $\mathcal{K} \backslash \mathcal{K}_{v}$ into a family $\tilde{\mathcal{K}}^{\prime}$ of vertices, edges and odd cycles in $\tilde{G}$. For this, interpret all the elements of $\mathcal{K} \backslash \mathcal{K}_{v}$ that do not meet $N(v)$ as a subgraph of $\tilde{G}$ and put them (with repetitions) in $\tilde{\mathcal{K}}^{\prime}$. For every occurrence of a vertex in $N(v)$ add $\{\tilde{v}\}$ to $\tilde{\mathcal{K}}^{\prime}$, and for every occurrence of an edge $r s$ with $s \in N(v)$ add the edge $r \tilde{v}$ to $\tilde{\mathcal{K}}^{\prime}$. For every cycle $C$ in $\mathcal{K} \backslash \mathcal{K}_{v}$ that is incident with a vertex in $N(v)$, the edge set $E(C)$ can be partitioned into the edge sets

\footnotetext{
${ }^{1}$ While this result was known before [6], it does not appear to have been published anywhere.
} 
of cycles in $\tilde{G}$. Add all the odd cycles to $\tilde{\mathcal{K}}^{\prime}$ and every second edge from every even cycle. This yields a family $\tilde{\mathcal{K}}^{\prime}$ of the same cost as $\mathcal{K} \backslash \mathcal{K}_{v}$ that covers every vertex in $V(\tilde{G}-\tilde{v})$ as often as $\mathcal{K} \backslash \mathcal{K}_{v}$, and which covers $\tilde{v}$ as often as $N(v)$ is covered in total by $\mathcal{K} \backslash \mathcal{K}_{v}$.

Thus the cost of $\tilde{\mathcal{K}}:=\tilde{\mathcal{K}}_{v} \cup \tilde{\mathcal{K}}^{\prime}$ is at most the cost of $\mathcal{K}$ minus $w_{v}$, that is, $\alpha_{w}(G)-w_{v}$. By $(2)$, this is at most $\alpha_{\tilde{w}}(\tilde{G})$. Hence, it only remains to show that $\tilde{\mathcal{K}}$ is a $\tilde{w}$-cover of $\tilde{G}$. By construction, every vertex $u \neq \tilde{v}$ is covered adequately by $\tilde{\mathcal{K}}$, so we only have to check how often we covered $\tilde{v}$. Clearly $\tilde{v}$ is covered by $\tilde{\mathcal{K}}$ at least as often as $\mathcal{K}$ covered $N(v)$ minus $\left|\mathcal{K}_{v}\right|$, since all we lose are the edges in $\mathcal{K}_{v}$, and for each cycle $C \in \mathcal{K}_{v}$ we observe that while $C$ covered two vertices in $N(v)$ its counterpart in $\tilde{\mathcal{K}}$ still covers $\tilde{v}$ once. As $\mathcal{K}$ covers $v$ exactly $w_{v}$ times, it follows that $\left|\mathcal{K}_{v}\right|=w_{v}$. Hence, $\tilde{\mathcal{K}}$ covers $\tilde{v}$ at least $d(v) \cdot \beta-w_{v}=\tilde{w}_{\tilde{v}}$ times, as desired.

\section{Minimally strongly $t$-imperfect graphs}

A graph $G$ is minimally (strongly) $t$-imperfect if it is (strongly) $t$-imperfect but every proper $t$-minor of $G$ is (strongly) $t$-perfect. An example of a minimally strongly $t$-imperfect graphs is $K_{4}$, which is also minimally $t$-imperfect. Thus, if a graph contains, for instance, $K_{4}$ as a $t$-minor then it is strongly $t$-imperfect as well as $t$-imperfect. This observation enabled a succinct characterisation of $t$-perfection in claw-free graphs [2], and will be helpful in what follows.

Theorem 2 lends credibility to the conjecture that $t$-perfection is always strong. One way to prove the conjecture would consist in verifying whether the minimally $t$-imperfect graphs coincide with the minimally strongly $t$-imperfect graphs. Unfortunately, a complete list of minimal elements is neither known for $t$-perfection nor for strong $t$-perfection.
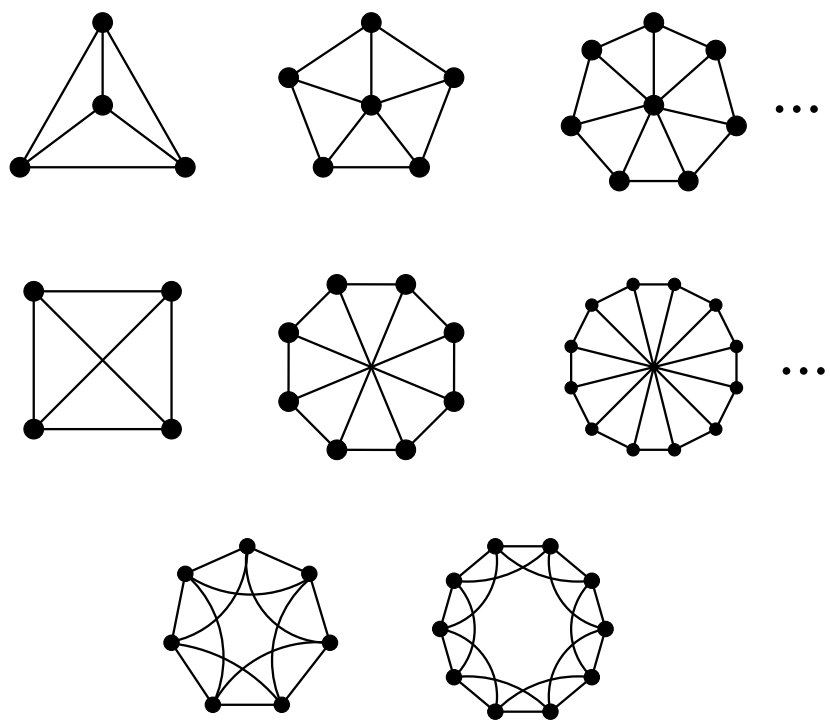

Figure 1: Minimally (strongly) $t$-imperfect graphs

Figure 1 shows some minimally $t$-imperfect graphs: the odd wheels and the 
even Möbius ladders (see Schrijver [14]), as well as two additional graphs, the squares $C_{7}^{2}$ and $C_{10}^{2}$ of the 7 -cycle and the 10-cycle (see [2]). All these graphs are minimally strongly $t$-imperfect as well.

In fact, that the odd wheels and the even Möbius ladders are minimally strongly $t$-imperfect can easily be deduced from the fact that almost bipartite graphs are strongly $t$-imperfect, which follows from Theorem 3. (A graph is almost bipartite if it can be made bipartite by deleting some vertex.)

The fact that $C_{7}^{2}$ and $C_{10}^{2}$ are minimally strongly $t$-imperfect will be proved in Lemma 5 below. We remark that these are the only squares of cycles that have this property.

One can obtain a few more minimally $t$-imperfect and minimally strongly $t$-imperfect graphs by adding any number of diagonals to $C_{10}^{2}[1]$. These graphs are depicted in Figure 2. To our knowledge, Figures 1 and 2 show all minimally (strongly) $t$-imperfect graphs known.
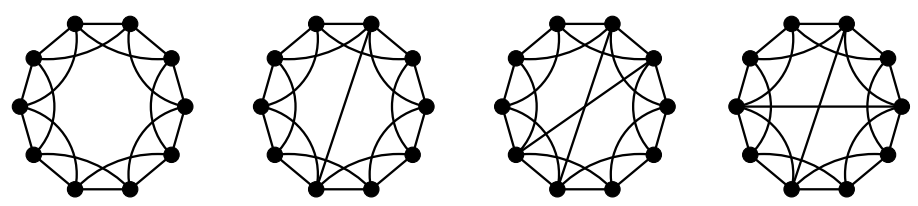

$C_{10}^{2}$
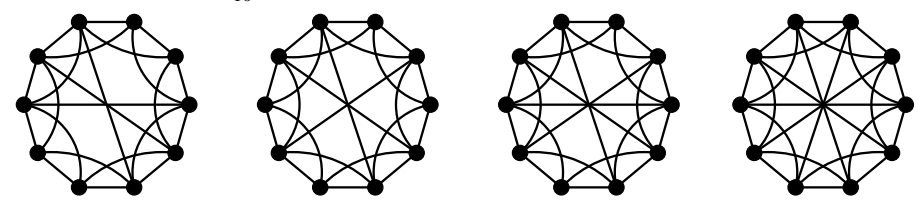

Figure 2: Some more minimally (strongly) $t$-imperfect graphs

Lemma 5. The graphs $C_{7}^{2}$ and $C_{10}^{2}$ are minimally strongly-t-imperfect.

Proof. As $C_{7}^{2}$ and $C_{10}^{2}$ are $t$-imperfect (consider for example the vector $x$ with $x_{v}=\frac{1}{3}$ for each vertex $v$ ), they are also strongly $t$-imperfect. Hence, since no vertex in $C_{7}^{2}$ and $C_{10}^{2}$ has independent neighbourhoods, it suffices to prove that for $j \in\{7,10\}$ the graph $C_{j}^{2}-v_{j}$ is strongly $t$-perfect. In both cases, $j=7$ and $j=10$, we proceed by induction on the total weight $w(V)$, where $V:=V\left(C_{j}^{2}-v_{j}\right)$ and $w$ is the given non-negative vector in $\mathbb{Z}^{V}$ for which we have to find a $w$-cover. As the case when $w(V)=0$ is trivial we will assume that $w$ is given with $w(V)>0$, and that the desired cover exists for all $w^{\prime}$ with $w^{\prime}(V)<w(V)$.

Recall that $\left\{v_{1}, \ldots, v_{j}\right\}$ are the vertices of $C_{j}$ in circular order, so that $v_{1}, v_{2}, v_{j-2}$ and $v_{j-1}$ have degree 3 in $C_{j}^{2}-v_{j}$. Denote by $\mathcal{S}$ the set of all stable sets of weight $\alpha_{w}:=\alpha_{w}\left(C_{j}^{2}-v_{j}\right)$, and write $w_{i}$ for $w\left(v_{i}\right)$.

First of all, if there is a triangle $T$ so that every $S \in \mathcal{S}$ meets $T$, then we define $w^{\prime}(v):=w(v)-1$ for $v \in T^{+}:=V(T) \cap \bigcup_{S \in \mathcal{S}} S$ and $w_{i}^{\prime}=w_{i}$ otherwise. As each $v \in T^{+}$has positive weight $w(v)$-otherwise $S \backslash\{v\}$ would be in $\mathcal{S}$ and miss $T$-we conclude that $w^{\prime}$ is non-negative. Since $T^{+} \neq \emptyset$ by assumption, the total weight $w^{\prime}(V)$ is smaller than $w(V)$. Hence, by induction there is a $w^{\prime}$-cover $\mathcal{K}^{\prime}$ of cost $\alpha_{w^{\prime}}$. Since $\alpha_{w^{\prime}}=\alpha_{w}-1$ the family $\mathcal{K}^{\prime} \cup T$ is a $w$-cover of cost $\alpha_{w}$, as desired. 
We can argue in a similar way if every $S \in \mathcal{S}$ meets the edge $v_{1} v_{j-1}$. So, let us assume from now on that for each triangle $T$ in $C_{j}^{2}-v_{j}$ there is a $S_{T} \in \mathcal{S}$ avoiding $T$, and that there exists an $S_{v_{1} v_{j-1}} \in \mathcal{S}$ that is disjoint from $\left\{v_{1}, v_{j-1}\right\}$.

For $C_{7}^{2}-v_{7}$, the stable set $S_{v_{4} v_{5} v_{6}}$ of weight $\alpha_{w}$ needs to consist of a single vertex $v_{k}$ with $k \in\{1,2,3\}$ as $v_{1} v_{2} v_{3}$ forms a triangle in $C_{7}^{2}-v_{7}$. Hence, $w_{k}=\alpha_{w}$. In the same way, we get that for some $l \in\{4,5,6\}$ the vertex $v_{l}$ has weight $\alpha_{w}$, too. Moreover, $v_{k}$ and $v_{l}$ have to be adjacent. If $(k, l)=(1,6)$, then all other vertices have weight 0 , and $\alpha_{w}$ times the edge $v_{1} v_{6}$ is a $w$-cover of $C_{7}^{2}-v_{7}$. On the other hand, if $k \in\{2,3\}$ and $l \in\{4,5\}$, then $w_{1}=w_{6}=0$. Furthermore, as $\left\{v_{2}\right\}=S_{v_{3} v_{4} v_{5}}$ and $\left\{v_{5}\right\}=S_{v_{2} v_{3} v_{4}}$ have weight $\alpha_{w}$, the stable set $\left\{v_{2}, v_{5}\right\}$ has weight $2 \alpha_{w}$, a contradiction.

Now, let us consider $C_{10}^{2}-v_{10}$. Let $K$ be a triangle in $C_{10}^{2}-v_{10}$, or let $K$ be the subgraph consisting of the edge $v_{1} v_{9}$. Suppose that $k \in V(K)$.

If $w(k)>0$ and $k$ has only one neighbour $s$ outside $K$ then, as $w\left(S_{K}\right)=\alpha_{w}$, $S_{K}$ contains $s$, since otherwise we could increase the weight of $S_{K}$ by including $k$. Since $S_{K} \backslash\{s\} \cup\{k\}$ is stable, it follows that $w(k) \leq w(s)$. Observe that this inequality trivially holds too, if $w(k)=0$. We use this rule to obtain a number of inequalities that are listed in the table below.

\begin{tabular}{c|c|c}
$K$ & $w(k) \leq w(s)$ & $w(k) \leq w(s)$ \\
\hline$v_{1} v_{2} v_{3}$ & (a) $w_{1} \leq w_{9}$ & (b) $w_{2} \leq w_{4}$ \\
$v_{7} v_{8} v_{9}$ & (c) $w_{9} \leq w_{1}$ & (d) $w_{8} \leq w_{6}$ \\
$v_{2} v_{3} v_{4}$ & (e) $w_{2} \leq w_{1}$ & \\
$v_{6} v_{7} v_{8}$ & (f) $w_{8} \leq w_{9}$ &
\end{tabular}

Now assume that the vertex $k \in V(K)$ has two adjacent neighbours $s$ and $t$ outside $K$ (and then no other neighbours outside $K$ ). Because $S_{K}$ can only contain one of $s$ and $t$, we deduce as above that $w(k) \leq \max \{w(s), w(t)\}$. Using this argumentation, we obtain

\begin{tabular}{c|l}
$K$ & $w(k) \leq \max \{w(s), w) t)\}$ \\
\hline$v_{3} v_{4} v_{5}$ & (g) $w_{3} \leq \max \left\{w_{1}, w_{2}\right\}$ \\
$v_{5} v_{6} v_{7}$ & (h) $w_{7} \leq \max \left\{w_{8}, w_{9}\right\}$ \\
$v_{1} v_{9}$ & (i) $w_{1} \leq \max \left\{w_{2}, w_{3}\right\}$ \\
$v_{1} v_{9}$ & (j) $w_{9} \leq \max \left\{w_{7}, w_{8}\right\}$
\end{tabular}

From (a) and (c), we get that $w_{1}=w_{9}$, and (g) together with (e) yields $w_{3} \leq w_{1}$. Symmetrically, we obtain $w_{7} \leq w_{9}$, and with (e), (f), (i) and (j) this results in

$$
\max \left\{w_{2}, w_{3}\right\}=w_{1}=w_{9}=\max \left\{w_{7}, w_{8}\right\} .
$$

By definition, $S_{v_{4} v_{5} v_{6}}$ avoids $v_{4} v_{5} v_{6}$ but has weight $\alpha_{w}$. Since $S_{v_{4} v_{5} v_{6}}$ meets both of the triangles $v_{1} v_{2} v_{3}$ and $v_{7} v_{8} v_{9}$ at most once we obtain from (3) that $w\left(S_{v_{4} v_{5} v_{6}}\right)=2 w_{1}$. Thus, (3) allows us to choose $s \in\left\{v_{7}, v_{8}\right\}$ and $s^{\prime} \in\left\{v_{2}, v_{3}\right\}$ so that $S:=\left\{v_{1}, s\right\}$ and $S^{\prime}:=\left\{v_{9}, s^{\prime}\right\}$ have weight $\alpha_{w}$.

Comparing the stable set $\left\{v_{1}, s, v_{4}\right\}$ to $S$ we get $w_{1}+w(s)+w_{4} \leq w(S)=$ $w_{1}+w(s)$ and thus $w_{4}=0$. Hence, $w_{2}=0$ too, by (b), and $w_{3}=w_{1}$, by (3). Symmetrically, comparing $\left\{v_{9}, s^{\prime}, v_{6}\right\}$ to $S^{\prime}$, we get that $w_{6}=w_{8}=0$.

To sum up, we have discovered that $w_{1}=w_{3}=w_{7}=w_{9}$ and that $w_{2}=$ $w_{4}=w_{6}=w_{8}=0$. Furthermore, $\alpha_{w}=w(S)=2 w_{1}$. Finally, as $\left\{v_{1}, v_{5}\right\}$ is stable, it follows that $w_{5} \leq w_{1}$. We conclude the proof by choosing a $w$-cover consisting of $w_{1}$ times the 5-cycle $v_{1} v_{3} v_{5} v_{7} v_{9}$ at a cost of $2 w_{1}$. 


\section{Strongly $t$-perfect claw-free graphs}

In order to prove Theorem 2, we only have to show that every claw-free strongly $t$-imperfect graph is $t$-imperfect. In fact, it suffices to prove this for all minimally strongly $t$-imperfect graphs $G$. Our first step in this direction is to show that $G$ is 3-connected:

Lemma 6. Let $G$ be a minimally strongly $t$-imperfect graph. If $G$ is claw-free then $G$ is 3-connected.

We postpone the lengthy proof of this lemma to the end of the section. Once equipped with Lemma 6 we may apply the following tool from [2].

Lemma 7. $[\mathbf{2}]^{2}$ Let $G$ be a 3-connected claw-free graph. If $G$ is t-perfect then one of the following statements holds true:

(i) $G$ is a line graph;

(ii) $G \in\left\{C_{6}^{2}-v_{1} v_{6}, C_{7}^{2}-v_{7}, C_{10}^{2}-v_{10}\right\}$.

We need one further ingredient for the proof of Theorem 2. The following theorem describes a TDI system for the matching polytope of a graph $G$ - this polytope is the convex hull in $\mathbb{R}^{E(G)}$ of the characteristic vectors of matchings in $G$. A graph $F$ is factor-critical if $F-v$ has a perfect matching for every $v \in V(F)$.

Theorem 8 (Cook [4]). For every graph $H$ the following system of inequalities is TDI:

$$
\begin{array}{cc}
y \in \mathbb{R}^{E(H)}, y \geq 0 & \text { for every } v \in V(H) \\
\sum_{e \in E(v)} y_{e} \leq 1 & \text { for every 2-connected factor-critical } F \subseteq H . \\
\sum_{e \in E(F)} y_{e} \leq\left\lfloor\frac{|V(F)|}{2}\right\rfloor
\end{array}
$$

We denote by $\mathbf{1}_{Z}$ the characteristic vector of the set $Z \subseteq V(G)$, where we abbreviate $\mathbf{1}_{\{z\}}$ by $\mathbf{1}_{z}$.

Proof of Theorem 2. We only need to show that a claw-free graph $G$ that is minimally strongly $t$-imperfect is also $t$-imperfect. By Lemma $6, G$ is 3 -connected. Thus, Lemma 7 is applicable and therefore, $G$ is either $t$-imperfect (as desired), or $G$ is a line graph, or $G \in\left\{C_{6}^{2}-v_{1} v_{6}, C_{7}^{2}-v_{7}, C_{10}^{2}-v_{10}\right\}$. Since $C_{7}^{2}$ and $C_{10}^{2}$ are minimally strongly $t$-imperfect by Lemma 5 , we only need to consider the cases when $G=C_{6}^{2}-v_{1} v_{6}$ or when $G$ is a line graph.

Suppose that $G=C_{6}^{2}-v_{1} v_{6}$, and pick a weight $w \in \mathbb{Z}^{V(G)}$ so that $G$ has no $w$-cover of cost $\alpha_{w}(G)$ of minimal total weight $w(V(G))$. Since $G$ is supposed to be minimally strongly $t$-imperfect it follows that $w>0$, i.e. that every entry of $w$ is positive. Then for $w^{\prime}:=w-\mathbf{1}_{v_{1} v_{2} v_{3}}$ there exists a $w^{\prime}$-cover $\mathcal{K}^{\prime}$ of cost $\alpha_{w^{\prime}}(G)$. However, every stable set of $S$ of weight $w(S)=\alpha_{w}(G)$ meets the triangle $v_{1} v_{2} v_{3}$, which implies that $\mathcal{K}^{\prime} \cup\left\{v_{1} v_{2} v_{3}\right\}$ is a $w$-cover of cost $\alpha_{w}(G)$, a contradiction.

\footnotetext{
${ }^{2}$ The lemma is the direct consequence of Lemma 9 in [2].
} 
So assume that $G$ is a line graph, of a graph $H$ say. First of all, note that $H$ has maximal degree $\leq 3$ since $G$, as a minimally strongly $t$-imperfect graph, cannot contain $K_{4}$ as a proper subgraph. Now, if the only 2-connected factorcritical subgraph $F$ of $H$ are odd cycles, then system (1) becomes (4) - which is TDI by Theorem 8 , a contradiction to the strong $t$-perfection of $G$. So, assume $H$ to contain a 2-connected factor-critical subgraph $F$ that is not an odd cycle. By a result of Lovász [10], $F$ then has a proper odd ear-decomposition, that is, there is a sequence of subgraphs $F_{0} \subseteq \ldots \subseteq F_{k}=F$, so that $F_{0}$ is an odd cycle and so that $F_{i+1}$ is obtained from $F_{i}$ by adding an odd $F_{i}$-path. Then, $F_{1} \subseteq F$ consists of three internally disjoint paths with common endvertices, so that exactly two of the paths have odd length. Viewed in $G$, the induced subgraph on the vertex set $E\left(F_{1}\right)$ can be $t$-contracted to $K_{4}$. Thus, $K_{4}$ is a $t$-minor of $G$, which shows that $G$ is $t$-imperfect.

The only missing link in our proof of Theorem 2 is Lemma 6 , i.e. the fact that every claw-free minimally strongly $t$-imperfect graph $G$ is 3 -connected. We will show this in two steps. In the first step, accomplished in Lemmas 9 13 , we ensure that $G$ is 2-connected and that every 2-separation (see the next paragraph) has a side that is a path. In the second step, for which we need Lemmas 14 and 15, we will prove that the minimum degree of $G$ is at least three.

For the first step we make use of the notion of $k$-separations. We say that $\left(G_{1}, G_{2}\right)$ is a separation of order $k$ of a graph $G$, or a $k$-separation of $G$, if $G_{1}, G_{2}$ are proper induced subgraphs of $G$ with $G=G_{1} \cup G_{2}$ and $\left|V\left(G_{1} \cap G_{2}\right)\right|=k$.

We use the following notation due to Gerards [7]. Let $\left(G_{1}, G_{2}\right)$ be a 2separation of an arbitrary graph $G$, and denote by $u$ and $v$ the two vertices contained in both $G_{1}$ and $G_{2}$. Given $w \in \mathbb{Z}^{V(G)}$, define $s_{w}^{i}(X)$ to be the maximum $w(S)$ among all stable sets $S$ in $G_{i}$ with $S \cap\{u, v\}=X$. If no confusion is possible we omit the subscript $w$. Moreover, we denote by $G_{i}+P_{2}$ the graph $G_{i}$ with an $u-v$ path of length 2 added, and by $G_{i}+P_{3}$ the graph $G_{i}$ plus an $u-v$ path of length 3. (Following Diestel [5] we denote the path on $k$ edges by $P_{k}$.)

The next two lemmas exclude already a good number of types of 2-separations in a minimally strongly $t$-imperfect graph. We mention that the lemmas do not appear explicitly in [7] but may, without effort, be extracted from the proof of Theorem 1.8.

Lemma 9 (Gerards [7]). Let $G$ be a graph, and let $\left(G_{1}, G_{2}\right)$ be a separation of order $\leq 2$. If $G_{1} \cap G_{2}$ forms a complete subgraph, and if $G_{1}$ and $G_{2}$ are strongly t-perfect, then $G$ is strongly t-perfect.

Lemma 10 (Gerards [7]). Let $G$ be a graph, and let $\left(G_{1}, G_{2}\right)$ be a 2-separation so that $V\left(G_{1}\right) \cap V\left(G_{2}\right)$ consists of two non-adjacent vertices $u$ and $v$. Then for every non-negative weight $w \in \mathbb{Z}^{V(G)}$ it holds thats:

(i) If $s^{2}(u, v)+s^{2}(\emptyset) \geq s^{2}(u)+s^{2}(v)$ and if $G_{1}+P_{2}$ as well as $G_{2}+P_{3}$ are strongly t-perfect then $G$ has a $w$-cover of cost $\alpha_{w}(G)$.

(ii) If $s^{2}(u, v)+s^{2}(\emptyset) \leq s^{2}(u)+s^{2}(v)$ and if $G_{1}+P_{3}$ as well as $G_{2}+P_{2}$ are strongly $t$-perfect then $G$ has a w-cover of cost $\alpha_{w}(G)$. 
Next, we relate the inequalities in (i) and (ii) in the previous lemma with the existence of odd or even induced $u-v$ paths.

Lemma 11. Let $\left(G_{1}, G_{2}\right)$ be a 2-separation of a graph $G$, and denote the two vertices common to both $G_{1}$ and $G_{2}$ by $u, v$. For every $w \in \mathbb{Z}^{V(G)}$ it holds that:

(i) If every induced $u-v$ path in $G_{2}$ has even length then $s^{2}(u, v)+s^{2}(\emptyset) \geq$ $s^{2}(u)+s^{2}(v)$.

(ii) If every induced $u-v$ path in $G_{2}$ has odd length then $s^{2}(u, v)+s^{2}(\emptyset) \leq$ $s^{2}(u)+s^{2}(v)$.

Proof. (i) Pick a stable set $S_{u}$ in $G_{2}$ with $u \in S_{u}$ but $v \notin S_{u}$ so that $w\left(S_{u}\right)=$ $s^{2}(u)$, and choose a stable set $S_{v}$ in $G_{2}$ with $v \in S_{v}, u \notin S_{v}$ and $w\left(S_{v}\right)=s^{2}(v)$. Denote by $K$ the vertex set of the component of $G_{2}\left[S_{u} \cup S_{v}\right]$ containing $u$. Then, as every induced $u-v$ path in $G_{2}$ has even length, it follows that $v \notin K$. The symmetric difference $S_{u} \triangle K$ is a stable set, and hence misses $\{u, v\}$, while the stable set $S_{v} \triangle K$ contains $\{u, v\}$. Since no vertex from $K$ lies in both of $S_{u}$ and $S_{v}$, we get

$$
s^{2}(u)+s^{2}(v)=w\left(S_{u}\right)+w\left(S_{v}\right)=w\left(S_{u} \triangle K\right)+w\left(S_{v} \triangle K\right) \leq s^{2}(\emptyset)+s^{2}(u, v) .
$$

(ii) We proceed in a similar way as in (i), only starting with stable sets $S_{\emptyset}$ and $S_{u, v}$ that miss, respectively contain, $\{u, v\}$.

For a 2-separation $\left(G_{1}, G_{2}\right)$ of a graph $G$, there is one case that is not addressed by Lemma 10, namely the case that every induced $u-v$ path in $G_{1}$ and in $G_{2}$ is even, or that every such path is odd.

Lemma 12. Let $\left(G_{1}, G_{2}\right)$ be a 2-separation of a graph $G$ so that $V\left(G_{1} \cap G_{2}\right)=$ $\{u, v\}$, and let $G_{1}$ and $G_{2}$ be strongly t-perfect. If every induced $u-v$ path in $G$ is even, or if every such path is odd, then also $G$ is strongly t-perfect.

Proof. Given a non-negative weight function $w: V(G) \rightarrow \mathbb{Z}$ we shall show for $i=1,2$ that there are non-negative weights $w^{i}: V(G) \rightarrow \mathbb{Z}$ with $w^{i} \mid V\left(G_{3-i}-\right.$ $\left.G_{i}\right)=0$ so that

(i) $w^{1}+w^{2}=w$, and

(ii) $\alpha_{w^{1}}\left(G_{1}\right)+\alpha_{w^{2}}\left(G_{2}\right) \leq \alpha_{w}(G)$.

This then establishes the lemma, as we can combine the $w^{i}$-covers of $G$ that are given by the strong $t$-perfection of the $G^{i}$ to a $w$-cover of $G$ of cost $\alpha_{w}(G)$.

In order to prove that such $w^{i}$ exist, we proceed by induction on the sum $w_{u}+w_{v}$. Clearly, if $w_{u}+w_{v}=0$, then the restrictions of $w$ to $G^{i}$ satisfy (i) and (ii). So assume w.l.o.g. that $w_{u}>0$, and set $\tilde{w}:=w-\mathbf{1}_{u}$, where $\mathbf{1}_{u}$ denotes the characteristic vector of $\{u\}$. By induction, we know that there exist $\tilde{w}^{1}$ and $\tilde{w}^{2}$ satisfying (i) and (ii).

In particular, there is a set $X \subseteq\{u, v\}$ such that $\alpha_{\tilde{w}_{1}}\left(G_{1}\right)=s_{\tilde{w}^{1}}^{1}(X)$ and $\alpha_{\tilde{w}_{2}}\left(G_{2}\right)=s_{\tilde{w}^{2}}^{2}(X)$. Now, if $\alpha_{\tilde{w}_{1}+\mathbf{1}_{u}}\left(G_{1}\right)=s_{\tilde{w}^{1}+\mathbf{1}_{u}}^{1}(X)$ then we may set $w_{1}:=$ $\tilde{w}^{1}+\mathbf{1}_{u}$ and $w^{2}:=\tilde{w}^{2}$ and are done. Hence we may assume that $\alpha_{\tilde{w}^{1}+\mathbf{1}_{u}}\left(G_{1}\right) \neq$ $s_{\tilde{w}^{1}+\mathbf{1}_{u}}^{1}(X)$. This can only happen if $u \notin X$, and if, moreover, there is a set $Y_{1} \subseteq\{u, v\}$ which contains $u$, such that $\alpha_{\tilde{w}^{1}}\left(G_{1}\right)=s_{\tilde{w}^{1}}^{1}\left(Y_{1}\right)$. (Then, we have that $\alpha_{\tilde{w}_{1}+\mathbf{1}_{u}}\left(G_{1}\right)=s_{\tilde{w}_{1}+\mathbf{1}_{u}}^{1}\left(Y_{1}\right)$.) Arguing in the same way for $\tilde{w}^{2}$, we find that 
there is a set $Y_{2} \subseteq\{u, v\}$ which contains $u$, such that $\alpha_{\tilde{w}^{2}}\left(G_{2}\right)=s_{\tilde{w}^{2}}^{2}\left(Y_{2}\right)$. By symmetry of $G_{1}$ and $G_{2}$, we may suppose that $Y_{1}=\{u\}$ and $Y_{2}=\{u, v\}$, since we are done if $Y_{1}=Y_{2}$.

So, depending on whether $X=\emptyset$ or $X=\{v\}$, we arrive at one of the following two cases:

(a) $\alpha_{\tilde{w}^{1}}\left(G_{1}\right)=s_{\tilde{w}^{1}}^{1}(\emptyset)=s_{\tilde{w}^{1}}^{1}(u)$ and $\alpha_{\tilde{w}^{2}}\left(G_{2}\right)=s_{\tilde{w}^{2}}^{2}(\emptyset)=s_{\tilde{w}^{2}}^{2}(u, v)$, or

(b) $\alpha_{\tilde{w}^{1}}\left(G_{1}\right)=s_{\tilde{w}^{1}}^{1}(v)=s_{\tilde{w}^{1}}^{1}(u)$ and $\alpha_{\tilde{w}^{2}}\left(G_{2}\right)=s_{\tilde{w}^{2}}^{2}(v)=s_{\tilde{w}^{2}}^{2}(u, v)$.

First, assume that case (a) holds. Now, if every induced $u-v$ path in $G$ is odd, then Lemma 11 (ii) implies that $\alpha_{\tilde{w}^{2}}\left(G_{2}\right)=s_{\tilde{w}^{2}}^{2}(u)=s_{\tilde{w}^{2}}^{2}(v)=s_{\tilde{w}^{2}}^{2}(\emptyset)=$ $s_{\tilde{w}^{2}}^{2}(u, v)$. Thus, setting $w^{1}:=\tilde{w}_{1}+\mathbf{1}_{u}$ and $w^{2}:=\tilde{w}^{2}$ will ensure (i) and (ii), as $s_{w^{1}}^{1}(u)=\alpha_{w^{1}}\left(G_{1}\right)$ and $s_{w^{2}}^{2}(u)=\alpha_{w^{2}}\left(G_{2}\right)$. So, in case (a), we may restrict our attention to the situation that every induced $u-v$ path in $G$ is even.

Then, by Lemma 11 (i), we have

$$
s_{\widetilde{w}^{1}}^{1}(v) \leq s_{\widetilde{w}^{1}}^{1}(u, v) .
$$

Furthermore, as we may otherwise set $w^{1}:=\tilde{w}_{1}$ and $w^{2}:=\tilde{w}^{2}+\mathbf{1}_{u}$, we see that

$$
s_{\tilde{w}^{2}}^{2}(u)<\alpha_{\tilde{w}^{2}}\left(G_{2}\right) .
$$

Set

$$
w^{1}:=\tilde{w}^{1}+\mathbf{1}_{v} \text { and } w^{2}:=\tilde{w}^{2}+\mathbf{1}_{u}-\mathbf{1}_{v} .
$$

Note that $\tilde{w}_{v}^{2}>0$ since $s_{\tilde{w}^{2}}^{2}(u)<\alpha_{\tilde{w}^{2}}\left(G_{2}\right)=s_{\tilde{w}^{2}}^{2}(u, v)$. By $(6)$, it is clear that $\alpha_{w^{2}}\left(G_{2}\right)=s_{w^{2}}^{2}(\emptyset)=s_{w^{2}}^{2}(u, v)$. On the other hand, (5) together with the fact that $s_{\tilde{w}^{1}}^{1}(u)=s_{\tilde{w}^{1}}^{1}(\emptyset)$ implies that $\alpha_{w^{1}}\left(G_{1}\right) \in\left\{s_{w^{1}}^{1}(\emptyset), s_{w^{1}}^{1}(u, v)\right\}$. Hence, our choice of $w^{1}$ and $w^{2}$ ensures (i) and (ii), as desired.

Now assume that case (b) above holds. If every induced $u-v$ path in $G$ is even, then Lemma 11 (i) implies that $\alpha_{\tilde{w}^{1}}\left(G_{1}\right)=s_{\tilde{w}^{1}}^{1}(\emptyset)=s_{\tilde{w}^{1}}^{1}(u, v)$. Thus, setting $w^{1}:=\tilde{w}_{1}$ and $w^{2}:=\tilde{w}^{2}+\mathbf{1}_{u}$ will ensure (i) and (ii). So, we will suppose from now on that every induced $u-v$ path in $G$ is odd.

By Lemma 11 (ii), we have

$$
s_{\widetilde{w}^{2}}^{2}(\emptyset) \leq s_{\widetilde{w}^{2}}^{2}(u),
$$

and (as we may otherwise set $w^{1}:=\tilde{w}_{1}+\mathbf{1}_{u}$ and $w^{2}:=\tilde{w}^{2}$ ) we see that

$$
s_{\tilde{w}^{1}}^{1}(u, v)<\alpha_{\tilde{w}^{1}}\left(G_{1}\right) \text { and } s_{\tilde{w}^{2}}^{2}(u)<\alpha_{\tilde{w}^{2}}\left(G_{2}\right) .
$$

Observe that $\tilde{w}_{v}^{2}>0$ by (8) and (b). Hence, setting

$$
w^{1}:=\tilde{w}^{1}+\mathbf{1}_{u}+\mathbf{1}_{v} \text { and } w^{2}:=\tilde{w}^{2}-\mathbf{1}_{v} .
$$

resolves our problem, as (8) implies that $\alpha_{w^{1}}\left(G_{1}\right)=s_{w^{1}}^{1}(u)=s_{w^{1}}^{1}(v)$, and $(7)$ implies that $\alpha_{w^{2}}\left(G_{2}\right) \in\left\{s_{w^{2}}^{2}(u), s_{w^{2}}^{2}(v)\right\}$.

We summarise the results obtained so far in the following lemma.

Lemma 13. Let $G$ be a minimally strongly t-imperfect graph. Then $G$ is 2connected, and if $\left(G_{1}, G_{2}\right)$ is a 2-separation of $G$ then one of $G_{1}$ and $G_{2}$ is a path. 
Proof. It follows from Lemma 9 that $G$ is 2-connected. Suppose that $G$ has a 2-separation $\left(H_{1}, H_{2}\right)$ with $V\left(H_{1}\right) \cap V\left(H_{2}\right)=\{u, v\}$. By Lemma 9, $u$ and $v$ are not adjacent.

If every induced $u-v$ path in $G$ is even or if every such path is odd then Lemma 12 implies that one of $H_{1}$ or $H_{2}$ is strongly $t$-imperfect, a contradiction, since $G$ is minimally strongly $t$-imperfect.

So we may assume that $H_{1}$ contains an even induced $u-v$ path, and $H_{2}$ contains an odd induced $u-v$ path. By minimality of $G$, this implies that $H_{1}+P_{3}$ and $H_{2}+P_{2}$ are strongly $t$-perfect. Now, pick a non-negative weight $w \in \mathbb{Z}^{V(G)}$ so that $G$ has no $w$-cover of cost $\alpha_{w}(G)$.

Applied to $\left(G_{1}, G_{2}\right):=\left(H_{1}, H_{2}\right)$, Lemma 11 (ii) together with Lemma 10 (ii) imply that $H_{2}$ also contains an even induced $u-v$ path. Moreover, Lemma 11 (i) and Lemma $10(\mathrm{i})$ applied to $\left(G_{1}, G_{2}\right):=\left(H_{2}, H_{1}\right)$ yield that $H_{1}$ contains an odd induced $u-v$ path. Hence, for all $i=1,2$ and $j=2,3$ the graph $H_{i}+P_{j}$ is a $t$ minor of $G$. For contradiction, assume that the $H_{i}+P_{j}$ are proper $t$-minors of $G$, and thus strongly $t$-perfect. Now, whichever value $s^{2}(u, v)+s^{2}(\emptyset)$ takes, either Lemma 10 (i) or (ii) is applicable in order to obtain the final contradiction.

We now turn to the second step in our proof of Lemma 6, that is, to proving that claw-free minimally strongly $t$-imperfect graphs have minimum degree at least three.

Lemma 14. Let $G=(V, E)$ be a graph, let $w \in \mathbb{Z}^{V}$, and assume $v$ to be a vertex with exactly two neighbours, $p$ and $q$. Let $p$ and $q$ be non-adjacent, and assume that $w_{p}=w_{v}=w_{q}$. Set $\tilde{G}=G / E(v)$, denote the new vertex by $\tilde{v}$ and define $\tilde{w} \in \mathbb{Z}^{V(\tilde{G})}$ by setting $\tilde{w}_{u}:=w_{u}$ for $u \in V(\tilde{G}-\tilde{v})$ and $\tilde{w}_{\tilde{v}}:=w_{v}$. If $\tilde{G}$ has a $\tilde{w}$-cover of cost $\alpha_{\tilde{w}}(\tilde{G})$ then $G$ has a w-cover of cost $\alpha_{w}(G)$.

Proof. Consider a stable set $\tilde{S}$ in $\tilde{G}$ with $\tilde{w}(\tilde{S})=\alpha_{\tilde{w}}(\tilde{G})$. If $\tilde{v} \in \tilde{S}$ then $S:=$ $(\tilde{S} \backslash\{\tilde{v}\}) \cup\{p, q\}$ is a stable set in $G$ with $w(S)=\alpha_{\tilde{w}}(\tilde{G})+w_{v}$. If, on the other hand, $\tilde{v} \notin \tilde{S}$ then $S:=\tilde{S} \cup\{v\}$ is stable in $G$, and $w(S)=\alpha_{\tilde{w}}(\tilde{G})+w_{v}$. Thus, we get

$$
\alpha_{\tilde{w}}(\tilde{G})+w_{v} \leq \alpha_{w}(G)
$$

By assumption, there is a $\tilde{w}$-cover $\tilde{\mathcal{K}}$ of $\tilde{G}$, which we may choose to cover $\tilde{v}$ exactly $\tilde{w}_{\tilde{v}}=w_{v}$ times. Observe that we may view $E(\tilde{G})$ as a subset of $E(G)$; for an edge $x \tilde{v}$ so that $x$ is a neighbour of $p$ as well as of $q$ we arbitrarily pick one of $x p$ and $x q$ and identify it with $x \tilde{v}$. Thus, viewed in $G$, the subfamily of $\tilde{\mathcal{K}}$ consisting of edges and odd cycles becomes a family of edges, odd cycles and odd $p-q$ paths; denote the latter subfamily of $\tilde{\mathcal{K}}$ by $\tilde{\mathcal{P}}$. By completing every $P \in \tilde{\mathcal{P}}$ to an odd cycle through $v$, and by replacing every occurrence of $\{\tilde{v}\}$ in $\tilde{\mathcal{K}}$ by one of $\{p\}$ and $\{q\}$ we obtain from $\tilde{\mathcal{K}}$ a family $\mathcal{K}^{\prime}$ of vertices, edges and odd cycles in $G$.

Set $\tilde{\gamma}:=|\tilde{\mathcal{P}}|$ and observe that as $\tilde{\mathcal{K}}$ covers $\tilde{v}$ exactly $w_{v}$ times, we get that $\tilde{\gamma} \leq w_{v}$. Moreover, it follows that each of $p$ and $q$ is covered by $\mathcal{K}^{\prime}$ at most $w_{v}$ times, while together they are covered $w_{v}+\tilde{\gamma}$ times since every $P \in \tilde{\mathcal{P}}$ leads to a cycle in $\mathcal{K}^{\prime}$ that meets $p$ as well as $q$. Since $v$ is contained in these cycles as well, it is covered $\tilde{\gamma}$ times. Hence, by adding $w_{v}-\tilde{\gamma}$ edges, $v p$ or $v q$, we can complete $\mathcal{K}^{\prime}$ to a $w$-cover $\mathcal{K}$. 
The cost of $\mathcal{K}$ is the cost of $\tilde{\mathcal{K}}$ plus the cost of extending the $P \in \tilde{\mathcal{P}}$ to cycles plus the cost of the additional edges incident with $v$. In other words, $\mathcal{K}$ costs

$$
\alpha_{\tilde{w}}(\tilde{G})+\tilde{\gamma}+w_{v}-\tilde{\gamma}=\alpha_{\tilde{w}}(\tilde{G})+w_{v} \leq \alpha_{w}(G),
$$

where the last inequality follows from (9).

The following lemma, which is quite similar to Lemma 20 in [2], uses an idea of Mahjoub [11]. For two vectors $w, w^{\prime} \in \mathbb{Z}^{V(G)}$ we write $w \leq w^{\prime}$ if $w_{v} \leq w_{v}^{\prime}$ for all $v \in V(G)$.

Lemma 15. Let $G$ be a graph, and let $w \in \mathbb{Z}^{V(G)}, w>0$, so that there is no $w$-cover of cost $\alpha_{w}(G)$ but for every $w^{\prime} \leq w$ with one strictly smaller entry there is a $w^{\prime}$-cover with cost $\alpha_{w^{\prime}}(G)$.

(i) If $G$ contains a path pvq so that $d(v)=2$ then $w_{v} \leq w_{q}$.

(ii) If $G$ contains a triangle prs and a neighbour $v \notin\{r, s\}$ of $p$ so that $d(p)=3$ then $w_{p} \leq w_{v}$.

Proof. Suppose there is an edge or triangle $X$ that is hit by every stable set $S$ of weight $w(S)=\alpha_{w}(G)$. Set $w^{\prime}:=w-\mathbf{1}_{X}$, and observe that $\alpha_{w^{\prime}}(G)=\alpha_{w}(G)-1$. Hence, by assumption there is a $w^{\prime}$-cover $\mathcal{K}^{\prime}$ of cost $\alpha_{w}(G)-1$, which together with $X$ yields a $w$-cover of cost $\alpha_{w}(G)$, a contradiction. This proves that for every edge or triangle $X$ there is a stable set $S_{X}$ of weight $\alpha_{w}(G)$ that misses $X$.

(i) Consider the stable set $S_{p v}$ of weight $\alpha_{w}(G)$ that misses the edge $p v$. Since $w_{v}>0$, it follows that $q \in S_{p v}$. Then $S:=S_{p v} \backslash\{q\} \cup\{v\}$ is a stable set with weight $w(S)=\alpha_{w}(G)-w_{q}+w_{v} \leq \alpha_{w}(G)$, which implies $w_{v} \leq w_{q}$, as desired.

(ii) Consider the stable set $S_{\text {prs }}$ of maximal weight that misses the triangle prs, and note that $v \in S_{\text {prs }}$. Then the stable set $S_{\text {prs }} \backslash\{v\} \cup\{p\}$ has weight $\alpha_{w}(G)-w_{v}+w_{p} \leq \alpha_{w}(G)$, which implies $w_{p} \leq w_{v}$, as desired.

We are finally prepared to prove Lemma 6 .

Proof of Lemma 6. By Lemma 13, we only need to convince ourselves that a minimally strongly $t$-imperfect graph $G=(V, E)$ does not contain any vertices of degree 2. So suppose otherwise, i.e. suppose that $G$ has vertices of degree 2 . As every cycle is strongly $t$-perfect, not all vertices may have degree 2 , and we may hence suppose that there is a path $P=u \ldots v$ in $G$ with all interior vertices of degree 2 in $G$ but with endvertices $u, v$ of higher degree. Furthermore, we may suppose that $P$ does indeed contain an interior vertex.

By Lemma 9 and the minimality of $G, u$ and $v$ cannot be adjacent. Since $K_{4}$ is strongly $t$-imperfect and $G$ minimal, $G$ cannot contain $K_{4}$ as a proper subgraph. Thus, it follows from the fact that $G$ is claw-free that both $u$ and $v$ have degree 3 and are incident with a triangle.

Among all non-negative $w \in \mathbb{Z}^{V}$ for which there is no $w$-cover of cost $\alpha_{w}(G)$ choose one, $w$ say, so that $w(V)$ is minimal. Since $G$ is strongly $t$-imperfect there is such a $w$ and, moreover, it holds that $w>0$ by the minimality of $G$. We may now apply Lemma 15 to the vertices in $P$ plus the two triangles incident with $u$ and $v$. This yields that $w$ is constant on $P$. Let $r$ be an interior vertex of $P$ and set $\tilde{G}:=G / E(r)$. Define $\tilde{w}$ as in Lemma 14 with $r$ in the role of $v$. Then, $\tilde{G}$ is a proper $t$-minor of $G$ and has thus a $\tilde{w}$-cover of cost $\alpha_{\tilde{w}}(\tilde{G})$. Now, however, 
Lemma 14 asserts that $G$ has a $w$-cover of $\operatorname{cost} \alpha_{w}(G)$, a contradiction to the choice of $w$.

\section{References}

[1] H. Bruhn and M. Stein, Unpublished.

$[2]$ , On claw-free t-perfect graphs, Preprint 2009.

[3] V. Chvátal, On certain polytopes associated with graphs, J. Combin. Theory (Series B) 18 (1975), 138-154.

[4] W. Cook, A minimal totally dual integral defining system for the b-matching polyhedron, SIAM J. Discrete Math. 4 (1983), 212-220.

[5] R. Diestel, Graph theory (3rd edition), Springer-Verlag, 2005.

[6] A.M.H. Gerards, personal communication.

[7] _ A min-max relation for stable sets in graphs with no odd- $K_{4}$, J. Combin. Theory (Series B) 47 (1989), 330-348.

[8] A.M.H. Gerards and F.B. Shepherd, The graphs with all subgraphs t-perfect, SIAM J. Discrete Math. 11 (1998), 524-545.

[9] D. Gijswijt and A. Schrijver, On the b-stable set polytope of graphs without bad $K_{4}$, SIAM J. Discrete Math. 16 (2003), 511-516.

[10] L. Lovász, A note on factor-critical graphs, Studia Scientiarum Mathematicum Hungarica 7 (1972), 279-280.

[11] A. R. Mahjoub, On the stable set polytope of a series-parallel graph, Math. Programming 40 (1988), 53-57.

[12] N. Sbihi and J.P. Uhry, A class of h-perfect graphs, Disc. Math. 51 (1984), 191-205.

[13] A. Schrijver, Strong t-perfection of bad- $K_{4}$-free graphs, SIAM J. Discrete Math. 15 (2002), 403-415.

[14] , Combinatorial optimization. Polyhedra and efficiency, SpringerVerlag, 2003.

[15] F. B. Shepherd, Applying Lehman's theorems to packing problems, Math. Prog. 71 (1995), 353-367.

Version 29th March 2010

Henning Bruhn <hbruhn@gmx.net> Department of Computer Science and System Analysis

Nihon University

Sakurajosui 3-25-40

Setagaya-Ku Tokyo 156-8550

Japan
Maya Stein <mstein@dim.uchile.cl> Centro de Modelamiento Matemático Universidad de Chile

Blanco Encalada, 2120

Santiago

Chile 